\section{The role of}

computerised tomography in predicting visual

\section{outcome in ocular trauma patients}

R Chaudhary ${ }^{1}$, M Upendran' ${ }^{1}$ N Campion²,

A Yeung ${ }^{1}$, R Blanch², P Morgan-Warren²,

I Gibb ${ }^{3}$, T Nelson ${ }^{4}$ and R Scott ${ }^{1,4}$

This article has been corrected since Advance Online Publication and a corrigendum is also printed in this issue

\begin{abstract}
Purpose Ocular blast injuries in the military setting are particularly associated with significant maxillofacial trauma and/or brain injury. The opportunity to perform a comprehensive ophthalmic evaluation is frequently limited in the acute multiple trauma scenario. We aim to describe the relationship between the clinical effects of acute ocular and orbital blast trauma with the findings on computerised tomography (CT). Methods This was a retrospective consecutive case series of all soldiers with facial and/or suspected ocular injuries. A total of 80 eyes that had suffered blast injuries of varying severity were studied. Assessment of orbital and ocular CT images were performed by military consultant radiologists. A comparison was made with actual clinical findings. Statistical analysis was performed using Fisher's exact test. Results No pathological findings were described in 37 of the 80 eyes imaged by orbital and ocular CT scans. Clinically, these eyes and orbits were all found to be intact, or had minor trauma. All foreign bodies and penetrating eye injuries were successfully diagnosed by CT. Absence of an orbital fracture did not rule out a globe injury. However, a corneal or scleral defect was less likely when an orbital fracture was absent. Conclusion The eye is a delicate structure prone to injury that requires urgent repair if breached. It is difficult to assess thoroughly in the unconscious or distressed patient. In this context, CT imaging is invaluable to be able to make a relatively confident prediction of clinical findings and decide upon the necessity for acute ophthalmic surgical intervention. Eye (2015) 29, 867-871; doi:10.1038/eye.2015.39; published online 27 March 2015
\end{abstract}

\section{Introduction}

Ocular trauma is one of the leading causes of visual loss in the working age population. ${ }^{1,2}$ It often affects young individuals and often causes permanent visual loss, with significant social and economic consequences. ${ }^{3}$ In the military setting, approximately $10 \%$ of war injuries with major trauma have eye injuries that are bilateral in $15-25 \%$ of cases. ${ }^{4,5}$ Ocular blast injuries are particularly associated with significant maxillofacial trauma and/or brain injury. ${ }^{6}$ Secondary blast injuries are the most common type of ocular blast injury, occurring in $\sim 42 \%$ of cases. This type of injury is from the impact of shrapnel from the explosive device itself or from exogenous debris propelled by the explosion. ${ }^{7}$

The opportunity to perform a comprehensive ophthalmic clinical evaluation is frequently limited in the acute multiple trauma scenario, as the patient is often in an intensive care unit bed, or anaesthetised. Ocular imaging has a major role in collecting clinical data, especially in the unconscious patient. A plain skull X-ray is performed to screen for cranial and facial fractures and will visualise radio-opaque foreign bodies. Computerised tomography (CT) scans are excellent at detection and localization of orbital and intraocular foreign bodies (IOFBs). ${ }^{8}$ Compared with other imaging modalities, it has also been found to be superior in detection of vitreous haemorrhage and lens dislocation. ${ }^{9}$

A CT scan will often diagnose other unsuspected intracranial and facial injuries. Ocular injury findings on CT correlate well with clinical findings. ${ }^{10}$

Other ancillary investigations include B-scan ultrasonography; an easy, cost-effective
${ }^{1}$ Department of Ophthalmology, Birmingham and Midland Eye Centre, City Hospital NHS Trust, Birmingham, UK

${ }^{2}$ Section of Neurotrauma and Neurodegeneration, School of Clinical and Experimental Medicine, University of Birmingham, Birmingham, UK

${ }^{3}$ Fort Blockhouse, Defence Centre for Imaging,

Gosport, UK

${ }^{4}$ Royal Centre for Defence Medicine, New Queen Elizabeth Hospital, Birmingham, UK

Correspondence: R Chaudhary, Department of Ophthalmology, Birmingham and Midland Eye Centre, City Hospital NHS Trust, Dudley Road, Birmingham B18 7QU, UK Tel: +44 (0)121 5543801 ; Fax: +44 (0)121507 5636 . E-mail: rchaudhary@ doctors.org.uk

Received: 22 September 2014

Accepted in revised form: 9 February 2015 Published online: 27 March 2015 
alternative to $\mathrm{CT}$ for the assessment of eye injuries. Ophthalmologists and emergency physicians can readily interpret the results of a B-scan. It has been shown to be superior to $\mathrm{CT}$ scanning in diagnosing retinal and choroidal detachments. ${ }^{11}$ It is particularly useful when ocular examination is obscured by opaque media. Care should be taken not to put pressure on the eye when examining a suspected ocular penetrating injury or globe rupture with an ultrasound probe.

Magnetic resonance imaging is contraindicated if there is any possibility of a ferromagnetic IOFB. Ferromagnetic foreign bodies produce large amounts of artifact that prevent meaningful images from being captured. The magnetic flux may cause them to move within the eye and cause further intraocular damage. ${ }^{12}$

Ocular trauma scoring systems such as the Ocular Trauma Score ${ }^{13}$ and the classification and regression tree $(\mathrm{CART})^{14}$ have been developed to predict visual outcome and prioritise ocular management within a multisystem trauma patient. These scoring systems include features often identifiable from CT scanning such as the presence of globe rupture, perforating injury, retinal detachment and the presence of an intraocular foreign body.

Mechanism of injury, extent of initial damage and initial visual acuity are the most important factors in predicting visual outcome. ${ }^{15}$ Patients that have a poor visual outcome or those requiring enucleation surgery have more CT findings. ${ }^{16}$

By defining the accuracy of CT imaging in eye trauma cases, it may be possible to diagnose certain ocular injuries and predict the visual prognosis without clinical examination, using current scoring systems. This has application in both civilian and combat blast injury scenarios and may help the ophthalmologist contribute to the multidisciplinary management strategy when it is impossible to physically examine the patient. CT scanning is relatively widespread in its availability compared to specialist ophthalmological services and the scans could be used to inform the telemedical management of injured patients in remote settings. This could potentially save valuable time and resources in ensuring that the patient has appropriate initial treatment and medical transportation. We aim to describe the relationship between the clinical effects of acute ocular and orbital blast trauma with the findings on CT.

\section{Materials and methods}

This was a retrospective consecutive case series of all soldiers with facial and/or suspected ocular injuries due to explosive blast suffered on active duty in Afghanistan. These patients often had complex multisystem trauma.

A CT scan was performed before clinical examination of the eyes by an ophthalmologist. A total of 40 male patients satisfying the criteria were admitted to Birmingham Hospitals between 2005 and 2009. A total of 80 eyes that had been exposed to explosive blast were studied.

Data collection included findings on emergency CT scanning performed as part of the preliminary investigation. The scans were performed on a Philips 16 slice but operating as a 6 slice to allow for redundancy on operations. The scans were $2 \mathrm{~mm}$ slices reconstructed every $1 \mathrm{~mm}$. Bone, brain, and soft tissue algorithms were used that allowed for more accurate assessment of the orbital walls and soft tissue contents as well as better delineation of foreign bodies. Assessment of orbital and ocular CT images was performed by military consultant radiologists at the Royal Centre for Defence Medicine, Birmingham. The assessment was performed systematically progressing from anterior to posterior looking for external soft tissue changes first, then assessing the anterior chamber, position of the lens, globe and posterior segment, bony orbit, foreign bodies, ocular vessels, and optic nerve as suggested by Kubal. ${ }^{17}$ Statistical analysis was performed using Fisher's exact test.

\section{Results}

Of 40 study patients, 11 had unilateral and 16 bilateral injuries reported after orbital and ocular CT scan imaging. No pathological findings were reported in 37 of the 80 eyes and adnexae. Where no pathological findings were reported, the eyes and adnexae were all found to be either intact; or had relatively minor closed globe injuries that did not require surgical intervention, such as corneal abrasion or hyphaema. With respect to the utility of CT scanning in the detection of injury requiring surgical intervention the $95 \%$ confidence interval for the positive predictive value of a normal CT result was $0.92-1.0$. The negative predictive value $95 \%$ confidence interval was $0.91-1.0$.

All foreign bodies were successfully diagnosed by CT scanning. Extraocular foreign bodies were found in 3 out of 80 eyes $(4 \%)$, an example of which can be seen in Figure 1 showing a $1.5 \mathrm{~mm}$ metallic corneal foreign body, confirmed on subsequent clinical examination. Fourteen $(17.5 \%)$ had intraocular foreign bodies (IOFB). All 18 penetrating injuries $(22.5 \%)$ were diagnosed on $\mathrm{CT}$, though 4 eyes did not have direct $\mathrm{CT}$ imaging evidence of a corneal or scleral (eyewall) defect, or a collapsed globe; but indirect evidence of globe penetration, including intraocular air and intraocular foreign bodies.

Intraocular air was present in 8 of 14 eyes with an IOFB $(57 \%)$; the relative risk for intraocular foreign body when air was present was 12 (95\% CI 5.58 to 25.82). Of the 18 eyes with a corneal or scleral defect, $12(66.7 \%)$ had intraocular air present on CT scan, and $6(33.3 \%)$ did not. Figure 2 shows a $2.4 \mathrm{~mm}$ metallic IOFB with orbital 


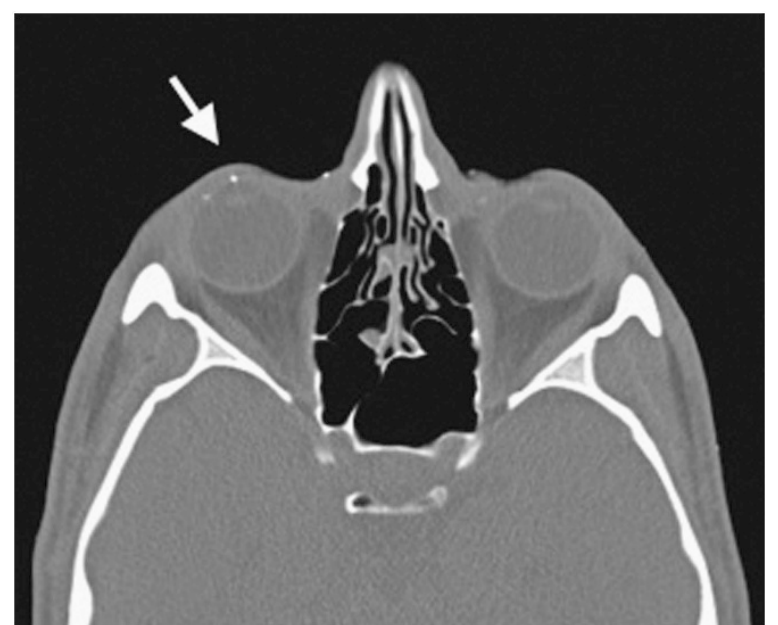

Figure 1 A $1.5 \mathrm{~mm}$ metallic corneal foreign body.

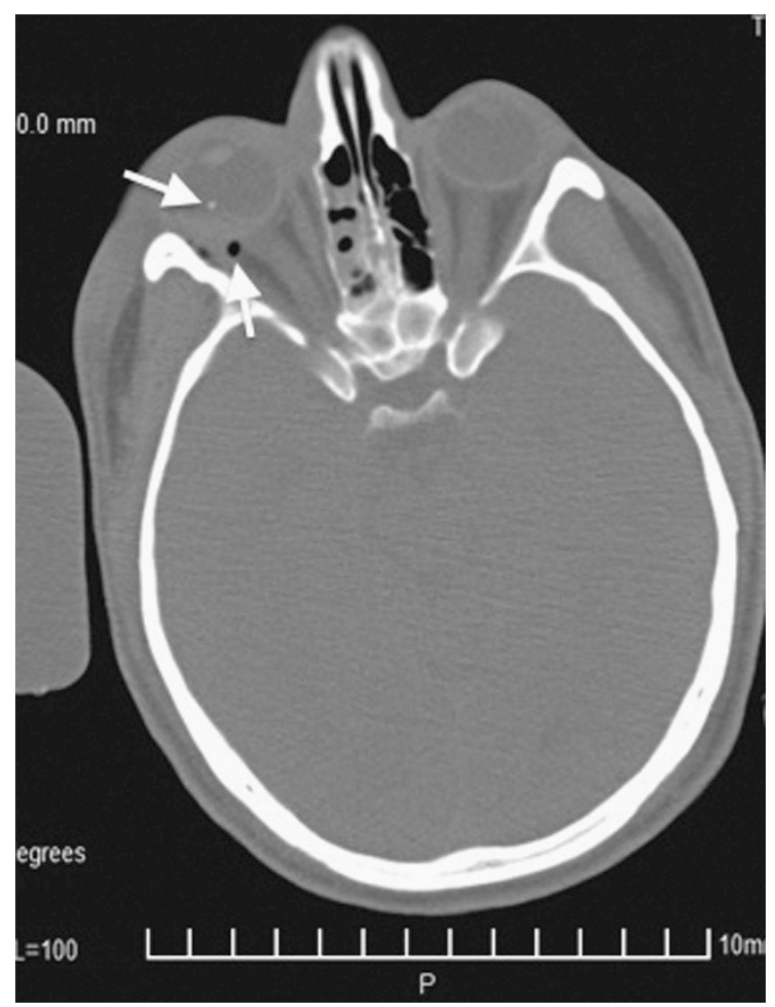

Figure 2 A $2.4 \mathrm{~mm}$ metallic IOFB with intraconal air.

intraconal air. As well as the IOFB, a further $2 \mathrm{~mm}$ intraorbital foreign body was reported close to the optic nerve in the optic canal. Clinically, the IOFB was confirmed, but the orbital foreign body was never identified, though subsequently there was severe traumatic optic neuropathy. Figure 3 shows anterior and posterior segment intraocular air; this patient had a $1.5 \mathrm{~mm}$ IOFB reported. Clinically, there was a penetrating

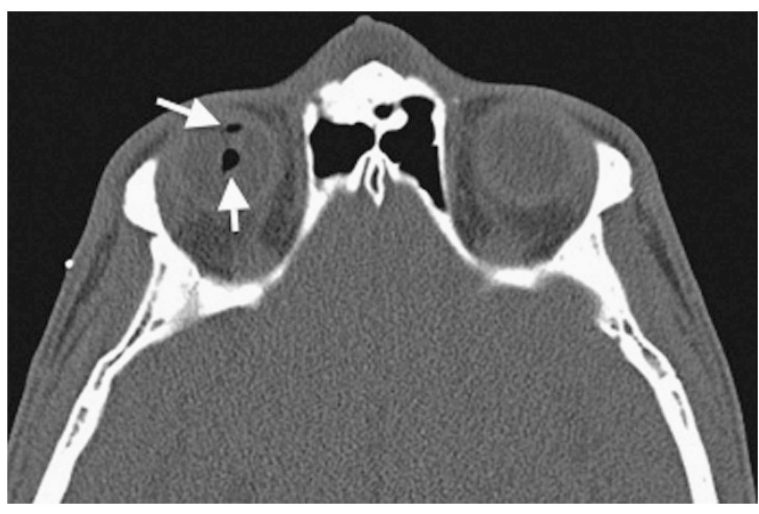

Figure 3 Anterior and posterior segment intraocular air.

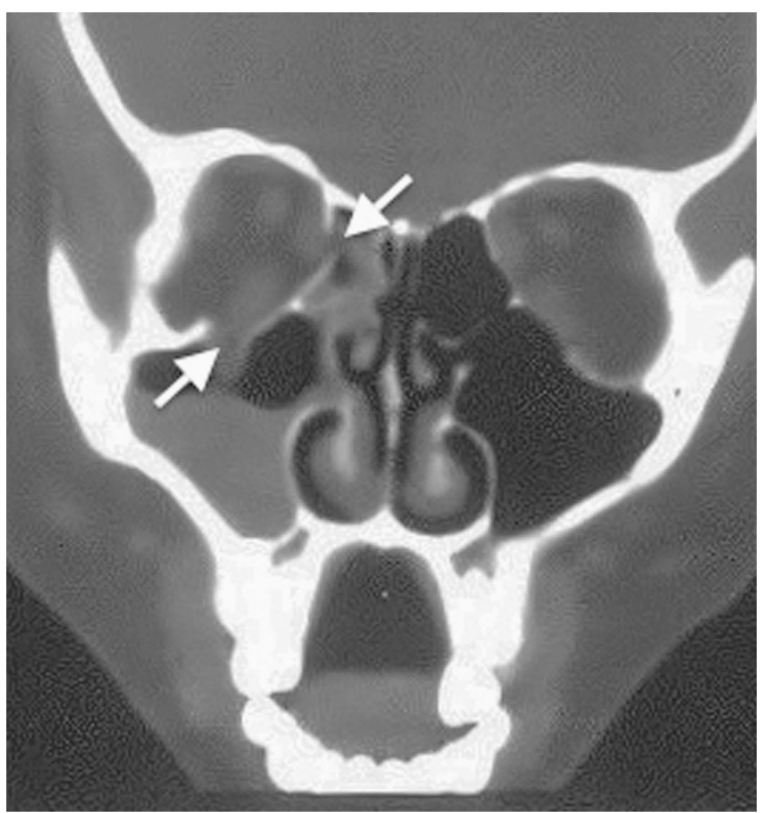

Figure 4 A medial orbital and floor fracture.

eye injury including a corneal (eyewall) penetrating injury, traumatic lens injury with cataract and an IOFB.

Absence of an orbital fracture did not rule out a globe injury. Nine eyes $(11 \%)$ had orbital fractures of which three had corneal or scleral defects (Fisher's exact test $P=0.48$ ); but a corneal or scleral defect was less likely when an orbital fracture was absent (negative predictive value $87 \%$ ). Figure 4 shows an example of a medial orbital and floor fracture. On examination, this patient had a scleral laceration on the same side. There was restriction of the ocular movements. Figure 5 shows a blood filled globe from a facial gunshot injury, with an open-globe injury, either an eyewall rupture or penetrating injury and a lateral orbital wall fracture. This picture was confirmed clinically. 


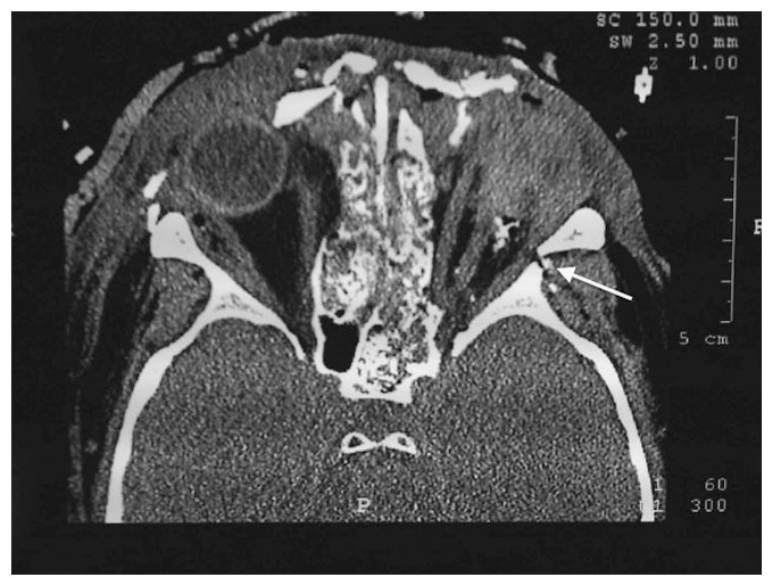

Figure 5 A blood-filled globe with either rupture or penetrating injury and a lateral orbital wall fracture from a facial gunshot injury.

\section{Discussion}

Management of patients with multisystem explosive blast injuries is often extremely challenging and requires a thorough and rapid assessment that directs prioritisation and logical ordering of clinical interventions. From our findings, a preliminary CT scan can give us clinically useful information especially when there is a lack of ophthalmic equipment or facilities.

Even relatively noticeable clinical findings such as traumatic enucleation can be difficult in these circumstances but clearly evident on CT imaging. The CT scan should include axial and coronal plane images with both soft tissue and bone windows. Our data suggest that an eye, which is intact on CT scan, is likely not to have a penetrating injury and immediate surgical intervention is probably not required. However, it is possible for there to be an eyewall defect that is undetected by CT scan. In our study $4 / 18(22 \%)$ had no CT evidence of an eyewall defect, though the rupture was inferred from secondary intraocular signs. Although a thorough clinical examination should not be omitted in the presence of a normal CT scan, it is likely that the globe is intact.

From our results, the presence of intraocular air on CT imaging is highly suggestive of serious injury. Intraocular air is also significant in cases where aeromedical evacuation is planned, depending on the initial altitude of the patient. Intraocular air depressurised to a normal cabin altitude pressure of 6-8000 ft from sea level, will expand by approximately $20-25 \%$. If there is sufficient gas in a closed eye, considered to be greater than $10 \%$ of the vitreous cavity volume, this can cause an acute rise in intraocular pressure. If the eye has an open wound and sufficient gas, the expansion has the potential to cause intraocular tissue extrusion. ${ }^{18}$ Where significant quantities of intraocular air have been diagnosed on CT scan aeromedical transfer at ground level cabin altitude pressure should be considered.

The eye is a delicate structure prone to injury that requires urgent repair if breached and is structure that is difficult to assess thoroughly in the unconscious or distressed patient, especially when there is injury causing swelling to the surrounding structures. In this situation it is invaluable to be able to make a relatively confident prediction of clinical findings and decide upon the necessity for acute ophthalmic surgical intervention from the results of imaging.

Studies have suggested that early IOFB removal reduces the risk of endophthalmitis. ${ }^{19}$ In contrast to this, it has been shown that there is no significant increase in risk of endophthalmitis in IOFB cases if treated early with a combination of broad-spectrum systemic and topical antibiotics with possible primary wound repair. In multiple injury cases, surgical delay is likely. Delayed IOFB removal has not been found to have a detrimental effect on visual outcome with early use of antibiotic treatment. ${ }^{20,21}$ Therefore early suspicion and detection with CT scanning potentially reduces the risk of endophthalmitis. The routine use of antibiotics in orbital fractures is controversial. The fear is of developing orbital cellulitis. Fractures adjacent to an infected sinus and nose blowing increase the risk of developing orbital cellulitis and potentially intracranial infection. Prophylactic antibiotics are advisable if adjacent sinusitis is present. ${ }^{22}$

Our findings are similar to those found in previous studies. In the absence of clinical information, CT imaging sensitivity and specificity has been reported as $75 \%$ and $93 \%$, respectively, for the prediction of open-globe injury. ${ }^{16}$ Literature searches show that there is an agreement that CT scanning is the gold standard for imaging in the majority of trauma cases. ${ }^{23} \mathrm{Kim} e t \mathrm{al}^{24}$ looked into the change of anterior chamber depth found on $\mathrm{CT}$ to aid in detecting an open-globe injury. This was found to be a helpful diagnostic criterion with a reported sensitivity of $92 \%$, specificity of $85 \%$, and diagnostic accuracy of $89 \%$. CT is useful in determining the size and location of a foreign body enabling the surgeon to plan the least traumatic method of removal. This is not possible with other imaging modalities. The main concern of CT scanning is exposure to ionizing radiation.

Radiation exposure from $\mathrm{CT}$ is higher than that from standard X-ray procedures, typically in a trauma scan. The increase in cancer risk from one CT scan is said to be small. The greater risk is that of inducing early cataracts, as the lens is much more radiosensitive than most other tissues. Many studies have shown an increased incidence of radiation-induced cataract amongst workers in radiologic departments and those that have undergone repeated CT scans. ${ }^{25}$ However, the clinical indication of performing a $\mathrm{CT}$ scan in this scenario outweighs the associated risks. 
It can be agreed that CT scans do not replace but complement clinical findings. Although our results reflect that CT images can provide us with reliable information, it cannot be solely relied upon for diagnosis in all openglobe injuries. Not all open-globe injuries will result in a foreign body or air detectable on CT; an open-globe injury may still be present even with negative CT findings. However, in the context that we describe CT imaging is particularly useful.

\section{Summary}

What was known before

- The opportunity to perform a comprehensive ophthalmic clinical evaluation is frequently limited in the acute multiple trauma scenario. Ocular trauma scoring systems have been developed to predict visual outcome and prioritise ocular management within a multisystem trauma patient. Ocular imaging has a major role in collecting clinical data, especially in the unconscious patient.

\section{What this study adds}

- A preliminary CT scan can give us clinically useful information in ocular trauma patients. An eye which is intact on CT scan is unlikely to have a penetrating injury and immediate surgical intervention is probably not required. The presence of intraocular air on CT imaging is highly suggestive of serious injury.

\section{Conflict of interest}

The authors declare no conflict of interest.

\section{Acknowledgements}

The study was carried out within the National Health Service (NHS). No extra financial support was sought or needed to complete the study. We would like to acknowledge the National Institute for Health Research Surgical Reconstruction and Microbiology Research Centre, Queen Elizabeth Hospital Birmingham.

\section{References}

1 Cheung CA, Rogers-Martel M, Golas L, Chepurny A, Martel JB, Martel JR. Hospital-based ocular emergencies: epidemiology, treatment, and visual outcomes. Am J Emerg Med 2013; 32(3): 221-224, pii: S0735-6757.

2 Wagner PJ, Lang GK. Mechanical ocular trauma. Curr Opin Ophthalmol 1996; 7(4): 57-64.

3 May DR, Kuhn FP, Morris RE, Witherspoon CD, Danis RP, Matthews GP et al. The epidemiology of serious eye injuries from the United States Eye Injury Registry. Graefes Arch Clin Exp Ophthalmol 2000; 238(2): 153-157.

4 Belkin M. Ocular war injuries in the Yom Kippur war. J Ocul Ther Surg 1983; 2: 40-49.
5 Gombos GM. Ocular war injuries in Jerusalem. Am J Ophthalmol 1969; 68: 474-478.

6 Breeze J, Allanson-Bailey LS, Hunt NC, Midwinter MJ, Hepper AE, Monaghan A et al. Surface wound mapping of battlefield occulo-facial injury. Injury 2012; 43: 1856-1860.

7 Scott RAH, Blanch RJ, Morgan-Warren PJ. Aspects of ocular war injuries. Trauma 2014; 17 (2): 83-92.

8 Woodcock MG, Scott RA, Huntbach J, Kirkby GR. Mass and shape as factors in intraocular foreign body injuries. Ophthalmology 2006; 113(12): 2262-2269.

9 Imran S, Amin S, Daula MIH. Imaging in Ocular Trauma Optimizing the Use of Ultrasound and Computerised Tomography. Pak J Ophthalmol 2011; 27(3): 146-151.

10 Lee HJ, Jilani M, Frohman L, Baker S. CT of orbital trauma. Emerg Radiol 2004; 10: 168-172.

11 Andreoli MT, Yiu G, Hart L, Andreoli CM. B-scan ultrasonography following open globe repair. Eye (Lond) 2014; 28(4): 381-385.

12 Dunkin JM, Crum VC, Swanger RS, Bokhari SAJ. Globe trauma. Semin Ultrasound CT MR 2011; 32: 51-56.

13 Kuhn F, Maisiak R, Mann L, Mester V, Morris R, Witherspoon CD. The ocular trauma score (OTS). Ophthalmol Clin North Am 2002; 15: 163-165.

14 Schmidt GW, Broman AT, Hindman HB. Vision survival after open globe injury predicted by classification and regression tree analysis. Ophthalmology 2008; 115: 202-209.

15 Ainbinder DJ, Mazzoli RA, Raymond WR, Hansen EA, Sanford EG. Ophthalmic care of the combat casualty, Ocular trauma scores. Section 2, chapter 6.

16 Joseph DP, Pieramici DJ, Beauchamp NJ. Computed tomography in the diagnosis and prognosis of open-globe injuries. Ophthalmology 2000; 107(10): 1899-1906.

17 Kubal WS. Imaging of orbital trauma. Radiographics 2008; 28(6): 1729-1739.

18 Lincoff H, Weinberger D, Reppucci V, Lincoff A. Air travel with intraocular gas. I. The mechanisms for compensation. Arch Ophthalmol 1989; 107: 902-906.

19 Chaudhry I, Shamsi F, Al-Harthi E, Al-Theeb A, Elzaridi E, Riley F. Incidence and visual outcome of endophthalmitis associated with intraocular foreign bodies. Graefes Arch Clin Exp Ophthalmol 2008; 246(2): 181-186.

20 Colyer M, Weber E, Weichel E, Dick JS, Bower KS, Ward TP et al. Delayed intraocular foreign body removal without endophthalmitis during Operations Iraqi Freedom and Enduring Freedom. Ophthalmology 2007; 114(8): 1439-1447.

21 Choovuthayakorn J, Hansapinyo L, Ittipunkul N, Patikulsila D, Kunavisarut P. Predictive factors and outcomes of posterior segment intraocular foreign bodies. Eye 2011; 25: 1622-1626.

22 Martin B, Ghosh A. Antibiotics in orbital floor fractures. Emerg Med J 2003; 20(1): 66.

23 Pinto A, Brunese L, Daniele S, Faggian A, Guarnieri G, Muto $\mathrm{M}$ et al. Role of computed tomography in the assessment of intraorbital foreign bodies. Semin Ultrasound CT MR 2012; 33(5): 392-395.

24 Kim SY, Lee JH, Lee YJ, Choi BS, Choi JW, In HS et al. Diagnostic value of the anterior chamber depth of a globe on CT for detecting open-globe injury. Eur Radiol 2010; 20(5): 1079-1084.

25 Chodick G, Bekiroglu N, Hauptmann M, Alexander BH, Freedman DM, Doody MM et al. Risk of cataract after exposure to low doses of ionizing radiation: a 20-year prospective cohort study among US radiologic technologists. Am J Epidemiol 2008; 168: 620. 